\title{
The Tutte polynomial characterizes simple outerplanar graphs
}

\author{
A. J. Goodall* \\ A. de Mier ${ }^{\dagger}$ \\ S. D. Noble $\ddagger$ \\ M. $\operatorname{Noy}^{\S}$
}

\begin{abstract}
We show that if $G$ is a simple outerplanar graph and $H$ is a graph with the same Tutte polynomial as $G$, then $H$ is also outerplanar. Examples show that the condition of $G$ being simple cannot be omitted.
\end{abstract}

\section{Introduction}

All graphs in this paper may have multiple edges and loops, unless otherwise stated. A loopless graph is 2-connected if and only if it has no cut vertices; a single loop is considered to be 2-connected, but no other graph with loops is. The blocks of a graph are its maximal 2-connected subgraphs.

We refer the reader to [4] for the definition and a detailed account of the properties of the Tutte polynomial. We mention here only the facts that are relevant to our work. The contraction-deletion rule states that if $e$ is an edge of $G$ then

$t(G ; x, y)= \begin{cases}t(G-e ; x, y)+t(G / e ; x, y), & \text { if } e \text { is neither a loop nor a bridge; } \\ y t(G-e ; x, y), & \text { if } e \text { is a loop; } \\ x t(G-e ; x, y), & \text { if } e \text { is a bridge. }\end{cases}$

The boundary condition to this recurrence is that $t(G ; x, y)=1$ when $G$ has no edges. It is clear from the recurrence that the coefficients of the Tutte polynomial are non-negative; we denote by $t_{i, j}(G)$ the coefficient of $x^{i} y^{j}$ in $t(G ; x, y)$. If $G$ has at least one edge, then $t_{0,0}(G)=0$.

We shall use the following properties of the Tutte polynomial.

(i) If $G$ has at least two edges then $t_{1,0}(G)=t_{0,1}(G)$.

*Department of Applied Mathematics and Institute of Theoretical Computer Science, Charles University, Malostranské nám. 25, 11800 Praha 1, Czech Republic. goodall.aj@googlemail.com

$\dagger^{\dagger}$ Departament de Matemàtica Aplicada II, Universitat Politècnica de Catalunya, Jordi Girona 1-3, 08034 Barcelona, Spain, anna.de.mier@upc.edu

${ }^{\ddagger}$ Department of Mathematical Sciences, Brunel University, Kingston Lane, Uxbridge UB8 3PH, U.K. steven.noble@brunel.ac.uk

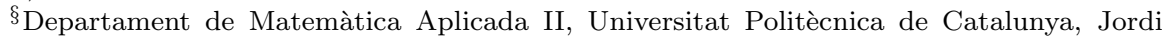
Girona 1-3, 08034 Barcelona, Spain, marc.noy@upc.edu 
(ii) A graph $G$, not a loop, is 2-connected if and only if $t_{1,0}(G) \geq 1$.

(iii) If $H$ is a minor of a 2-connected graph $G$ then $t_{i, j}(H) \leq t_{i, j}(G)$.

(iv) The irreducible factors of $t(G ; x, y)$ over $\mathbb{Z}[x, y]$ correspond precisely to the Tutte polynomials of the blocks of $G$.

Statements (i) and (ii) are well-known facts, easily proved by induction; (iii) was noticed by Brylawski [3, Corollary 6.9]; (iv) was proved in [8]. The coefficient $t_{1,0}(G)$ is known as Crapo's beta invariant, denoted by $\beta(G)$.

A 2-connected graph is series-parallel if it can be obtained from $K_{2}$ by subdividing and doubling edges; an arbitrary graph is series-parallel if all its blocks are series-parallel. Equivalently, a loopless graph is series-parallel if it does not contain $K_{4}$ as a minor [6]. It was proved in [2] that a 2-connected graph is series-parallel if and only if $\beta(G)=1$. Let us recall the simple proof of this fact. If $G$ is simple, series-parallel, 2-connected and has at least three vertices, then it has a vertex $x$ of degree two. Let $e$ and $f$ be the edges incident with $x$. Then $G / e$ is series-parallel and 2-connected, and by induction $\beta(G / e)=1$. On the other hand, $G-e$ has $f$ as a bridge, hence $\beta(G-e)=0$. By the contraction-deletion rule, we deduce that $\beta(G)=1$. The base case of the induction is a single edge $K_{2}$. If $G$ has multiple edges, it follows easily from the contraction-deletion rule that $\beta(G)$ is the value of $\beta$ of the simplification of $G$ (i. e., the graph obtained from $G$ by replacing every multiple edge by a single edge). Conversely, assume $G$ is a 2-connected garph that is not series-parallel. Then $G$ contains a minor $H$ isomorphic to $K_{4}$. By statement (iii) above, this implies $\beta(G) \geq \beta\left(K_{4}\right)$. Since $\beta\left(K_{4}\right)=2$, we are done.

The previous results, together with the fact that a graph is series-parallel if and only if its blocks are series-parallel, imply the following: if $G$ is seriesparallel and $t(G ; x, y)=t(H ; x, y)$, then $H$ is also series-parallel. In other words, the Tutte polynomial characterizes the class of series-parallel graphs. (It does not characterize series-parallel graphs individually, since a Whitney twist can be used to change a graph but not its Tutte polynomial.)

The goal of this paper is to prove a similar result for outerplanar graphs. Recall that a graph is outerplanar if it can be drawn in the plane in such a way that all the vertices are on the outer face. A 2-connected outerplanar graph with at least three vertices has a unique Hamilton cycle, which can be chosen to bound the outer face: for such a graph, an edge is called external if it lies on the boundary of the outer face, and internal otherwise. A graph is outerplanar if it does not contain $K_{4}$ or $K_{2,3}$ as a minor [5]. Notice that loopless outerplanar graphs form a subclass of series-parallel graphs, and that a graph is outerplanar if and only if its blocks are outerplanar.

Theorem 1. If $G$ is a simple outerplanar graph and $t(G ; x, y)=t(H ; x, y)$, then $H$ is also outerplanar.

The proof is given in Section 3, and is based on characterizing outerplanar graphs by means of the coefficients of degree two in $t(G ; x, y)$ (see Theorem 5 ), while for series-parallel graphs the linear coefficients were sufficient. It must be 
stressed that the condition of $G$ being simple is essential, otherwise the result is not true, as shown later by the examples in Section 4. This is in contrast with the characterization of series-parallel graphs, where simplicity is not needed. Let us also remark that the Tutte polynomial does not characterize simple outerplanar graphs individually: there are pairs of simple non-isomorphic outerplanar graphs sharing the same Tutte polynomial (see [10]); moreover, they have different cycle matroids, i. e., one cannot be obtained from the other by Whitney twists.

Generally speaking, classes of graphs defined in terms of forbidden minors are not characterized by the Tutte polynomial. For example there are planar graphs that have the same Tutte polynomial as a non-planar graph (see [9]). Thus, it is interesting to note the following consequence of our result. We denote by $\operatorname{Ex}(H)$ the class of graphs not containing $H$ as a minor. A 2-connected, simple graph in $\operatorname{Ex}\left(K_{2,3}\right)$ is either outerplanar or isomorphic to $K_{4}([1])$. Since $K_{4}$ is uniquely characterized by its Tutte polynomial, it follows from the previous arguments that if $G$ is a simple graph in $\operatorname{Ex}\left(K_{2,3}\right)$ and $t(G ; x, y)=t(H ; x, y)$, then $H$ is also in $\operatorname{Ex}\left(K_{2,3}\right)$.

We shall briefly return to the topic of classes of graphs characterized by the Tutte polynomial in the final section, the rest of the paper being devoted to the proof of Theorem 1.

\section{Open ear decompositions}

An open ear decomposition of a graph $G$ is a partition of the edges of $G$ into a sequence of simple paths $P_{0}, P_{1}, \ldots, P_{s}$ such that $P_{0}$ is a single edge, each endpoint of $P_{i}, 1 \leq i \leq s$, is contained in some $P_{j}, j<i$, and the internal vertices of $P_{i}$ are not vertices of any other $P_{j}, j<i$. The $P_{i}$ are called the ears of the decomposition. A graph has an open ear decomposition if and only if it is 2-connected [12]. The length $s$ of an open ear decomposition of $G$ equals its nullity or cyclomatic number, i. e., $s=|E|-|V|+1$.

An ear $P_{i}$ is nested in $P_{j}, j<i$, if both endpoints of $P_{i}$ belong to $P_{j}$. The nest interval of $P_{i}$ in $P_{j}$ is the path in $P_{j}$ between the two endpoints of $P_{i}$. An open ear decomposition is nested if

(i) For each $i>1$ there is some $j<i$ such that $P_{i}$ is nested in $P_{j}$,

(ii) If two ears $P_{i}$ and $P_{i^{\prime}}$ are nested in the same ear $P_{j}$ then either the nest interval of $P_{i}$ contains that of $P_{i^{\prime}}$ or vice versa, or the two nest intervals are disjoint.

Note that two ears that prevent an ear decomposition from being nested create a $K_{4}$ minor. Indeed, we have the following result:

Lemma 2. [7] A 2-connected graph $G$ is series-parallel if and only if every open ear decomposition of $G$ is nested.

A useful corollary for us is the following lemma. 
Lemma 3. A simple 2-connected series-parallel graph $G \neq K_{2}$ admits an open ear decomposition in which the last ear contains at least two edges.

Proof. The proof is by induction on the number of edges of $G$. If $G$ is a triangle then the result is true. Otherwise since $G$ is simple there exists a vertex $v$ incident with just two edges $e$ and $f$. If $e$ and $f$ form a triangle with edge $g$ then $G-e-f$ is 2 -connected. So $G-e-f$ has an open ear decomposition and $e, f$ can be added as the last ear to form an open ear decomposition as required. On the other hand if $e$ and $f$ are not part of a triangle then $G / e$ is simple, 2-connected and series parallel, and so by the inductive hypothesis, $G / e$ has an open ear decomposition with the last ear having at least two edges. This can be modified to give an open ear decomposition of $G$ by adding $e$ to the ear containing $f$.

The last ear $P$ in an open ear decomposition of $G$ is an induced path. Let $G \backslash P$ denote the minor of $G$ obtained by deleting all the edges in $P$, and $G / P$ the minor obtained by successively contracting all the edges in $P$ (in any order).

The following lemma is elementary and we omit its proof.

Lemma 4. If $P$ is the last path in an open ear decomposition of $G$ and has $k$ edges then

$$
t(G ; x, y)=\left(x^{k-1}+\cdots+x+1\right) t(G \backslash P ; x, y)+t(G / P ; x, y) .
$$

\section{Simple outerplanar graphs}

In this section we prove that the Tutte polynomial characterizes the class of simple outerplanar graphs.

Theorem 5. Let $G \neq K_{2}$ be a simple, 2-connected, series-parallel graph. Then

$$
t_{2,0}(G) \geq t_{0,2}(G)+1
$$

with equality if and only if $G$ is outerplanar. Moreover, when $G$ is outerplanar $t_{2,0}(G)$ is equal to the number of inner faces of $G$.

Proof. The $k$-cycle $C_{k}, k \geq 3$, is outerplanar. Here $t_{2,0}\left(C_{k}\right)=1$ and $t_{0,2}\left(C_{k}\right)=$ 0 , so equality holds in equation (2). Assume inductively that the theorem holds for every simple, 2-connected, series-parallel graph with nullity at most $s$ (open ear decompositions of length at most $s$ ). Since the theorem is true for all cycles, we can take $s \geq 1$. Given a simple, 2-connected, series-parallel graph $G$ with nullity $s+1$, let $P$ be the last ear in a given open ear decomposition of $G$. By Lemma 3 we can assume $P$ has at least two edges.

The graph $G \backslash P$ is 2-connected since it has an open ear decomposition. If $G / P$ were also 2-connected, Lemma 4 and property (ii) of the Tutte polynomial would imply that $\beta(G) \geq 2$, which is contrary to $G$ being series-parallel. Thus $G / P$ has at least two blocks, none of which are bridges, since by contracting an edge in a bridgeless graph one cannot obtain a bridge. The graph $G / P$ has a 
loop if and only if the endpoints of $P$ are the endpoints of an edge of $G$, and there can be no other loops other than this one.

The graph $G \backslash P$ is a simple, 2-connected, series-parallel graph with nullity $s$. By the inductive hypothesis, $t_{2,0}(G \backslash P) \geq t_{0,2}(G \backslash P)+1$ with equality if and only if $G \backslash P$ is outerplanar. Note that if $G$ is outerplanar then $G \backslash P$ is also outerplanar.

By equation (1) together with $t_{1,0}(G \backslash P)=1$, we have

$$
t_{2,0}(G)=t_{2,0}(G \backslash P)+1+t_{2,0}(G / P),
$$

where

$$
t_{2,0}(G / P)=\left\{\begin{array}{ll}
1 & \text { if } G / P \text { has two blocks, neither a loop } \\
0 & \text { otherwise. }
\end{array} .\right.
$$

Equation (1) also gives

$$
t_{0,2}(G)=t_{0,2}(G \backslash P)+t_{0,2}(G / P) .
$$

Since $G / P$ cannot have any bridges, by property (i) of the Tutte polynomial the coefficient of $y$ in all of its blocks is equal to 1 , and hence

$$
t_{0,2}(G / P)= \begin{cases}1 & \text { if } G / P \text { has two blocks } \\ 0 & \text { otherwise }\end{cases}
$$

Equations (3) and (4) now imply

$$
\begin{aligned}
t_{2,0}(G)-t_{0,2}(G)-1 & =\left[t_{2,0}(G \backslash P)-t_{0,2}(G \backslash P)-1\right]+1+t_{2,0}(G / P)-t_{0,2}(G / P) \\
& \geq 1+t_{2,0}(G / P)-t_{0,2}(G / P),
\end{aligned}
$$

where the inequality follows by the inductive hypotheses on $G \backslash P$. There is equality if and only if $G \backslash P$ is outerplanar.

Since

$$
1+t_{2,0}(G / P)-t_{0,2}(G / P)= \begin{cases}0 & \text { if } G / P \text { has two blocks, one a loop } \\ 1 & \text { otherwise }\end{cases}
$$

we obtain

$$
t_{2,0}(G)-t_{0,2}(G)-1 \geq 0
$$

with equality if and only if $G \backslash P$ is outerplanar and $G / P$ has two blocks, one a loop. The latter can be achieved if and only if $P$ has the same endpoints as an external edge of $G \backslash P$ (in which case $G$ is also outerplanar). Indeed, if the endpoints of $P$ are those of an internal edge of the outerplanar graph $G \backslash P$, then $G / P$ has three blocks including the loop. In this case adding $P$ to $G \backslash P$ creates a $K_{2,3}$ minor, so that $G$ is not outerplanar. If $P$ has endpoints on the external face at distance more than one apart then $G / P$ has no loops, and in this case $G$ is not outerplanar, the addition of $P$ to $G \backslash P$ again forming a $K_{2,3}$ minor. 
We conclude that $t_{2,0}(G) \geq t_{0,2}(G)+1$ with equality if and only if $G$ is outerplanar, and this completes the induction step.

Finally we prove that when $G$ is a simple, 2-connected, outerplanar graph, $t_{2,0}(G)$ is equal to the number of interior faces, equivalently, the nullity of $G$. Alternatively, $t_{0,2}(G)$ is equal to the number of internal edges of $G$.

The statement is true for the cycles $C_{k}, k \geq 3$, that have no internal edges. Take inductively a simple, 2-connected, outerplanar graph $G$ and let $e$ be one of its internal edges. Since $t_{0,2}(G)=t_{0,2}(G \backslash e)+t_{0,2}(G / e)$, and $G / e$ has precisely two blocks, neither of which is a bridge (or loop), so that $t_{0,2}(G / e)=1$, the result now follows by induction.

Theorem 1 is a direct consequence of Theorem 5 .

Brylawski's relations [3, Theorem 6.6] are a set of linear equations satisfied by the coefficients of the Tutte polynomial. One of them states that

$$
t_{0,2}(G)-t_{1,1}(G)+t_{2,0}(G)=t_{1,0}(G)
$$

if $G$ has at least three edges. (Property (i) in the introduction is also one of the relations.) From the previous theorem we obtain as a corollary:

Corollary 6. Let $G \neq K_{2}$ be a simple, 2-connected, series-parallel graph. Then

$$
2 t_{2,0}(G)-2 \geq t_{1,1}(G),
$$

with equality if and only if $G$ is outerplanar.

\section{Concluding remarks}

The graphs $G$ and $H$ on the left of Figure 1 have the same Tutte polynomial, equal to

$$
\begin{aligned}
x^{5}+ & 2 x^{4} y+2 x^{3} y^{2}+2 x^{2} y^{3}+2 x y^{4}+y^{5}+3 x^{4}+7 x^{3} y+8 x^{2} y^{2}+7 x y^{3}+3 y^{4} \\
& +4 x^{3}+9 x^{2} y+9 x y^{2}+4 y^{3}+3 x^{2}+5 x y+3 y^{2}+x+y .
\end{aligned}
$$

The first one is outerplanar, while the second one is not. This shows that the condition of $G$ being simple in Theorem 1 cannot be omitted. This example can be generalized to the infinite families $G_{n}$ and $H_{n}$, shown in Figure 1, where $n$ is the number of triangles to the left of the central quadrilateral. To prove that they have the same Tutte polynomial, we argue by induction on $n$. First notice that $G_{1}=G$ and $H_{1}=H$. If $G_{n}^{*}$ and $H_{n}^{*}$ are as on the right of Figure 1, then by the contraction-deletion rule we have

$$
\begin{aligned}
& t\left(G_{n}\right)=x t\left(G_{n-1}\right)+t\left(G_{n-1}^{*}\right), \\
& t\left(G_{n}^{*}\right)=y t\left(G_{n-1}^{*}\right)+t\left(G_{n}\right) .
\end{aligned}
$$

We write $t(G)$ instead of $t(G ; x, y)$ for simplicity. From the relations above it is not difficult to check that

$$
t\left(G_{n}\right)=(x+y+1) t\left(G_{n-1}\right)-x y t\left(G_{n-2}\right) .
$$


G
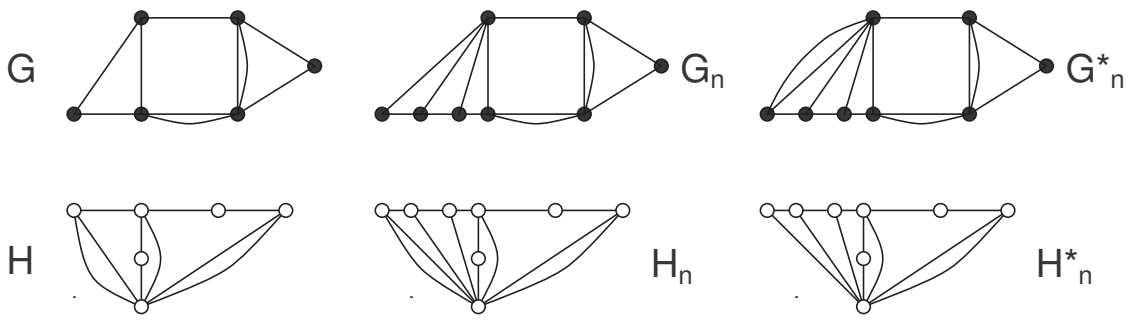

Figure 1: Non-outerplanar graphs with the same Tutte polynomial as outerplanar graphs.

In the same way we obtain

$$
t\left(H_{n}\right)=(x+y+1) t\left(H_{n-1}\right)-x y t\left(H_{n-2}\right) .
$$

One can check that $t\left(G_{2}\right)=t\left(H_{2}\right)$, and since $t\left(G_{1}\right)=t\left(H_{1}\right)$, the result follows by induction.

One may wonder which other natural classes of graphs $\mathcal{G}$ have the property that whenever $G$ is a member of $\mathcal{G}$ and $t(G ; x, y)=t(H ; x, y)$ for some graph $H$, then $H$ is also in $\mathcal{G}$. As the chromatic polynomial is an evalution of the Tutte polynomial, graphs whose chromatic number is at most $k$ form one such class. For instance, bipartite graphs are precisely those that satisfy $t(G ;-1,0) \neq 0$.

A possibility is to look at minor-closed classes of graphs. From now on all graphs are assumed to be simple. The easiest example are forests, the graphs in $\operatorname{Ex}\left(K_{3}\right)$, whose Tutte polynomials are of the form $x^{k}$ for some $k$. As mentioned in the introduction, the class $\operatorname{Ex}\left(K_{2,3}\right)$ is also characterized by the Tutte polynomial, but planar graphs are not.

Let $\mathcal{G}=\operatorname{Ex}(\mathcal{F})$ be the class of graphs not containing any of the graphs in $\mathcal{F}$ as a minor. If all the graphs in $\mathcal{F}$ are 2-connected, then a graph $G$ belongs to $\mathcal{G}$ if and only if the blocks of $G$ belong to $\mathcal{G}$. For a characterization of a class with this property by the Tutte polynomial, statement (iv) in the introduction implies that it suffices to consider the 2-connected members of $\mathcal{G}$.

Let $C_{4}^{+}$denote the 4 -cycle with a chord. The 2-connected members of the class $\operatorname{Ex}\left(C_{4}^{+}\right)$are $K_{2}$ and $C_{k}, k \geq 3$, each of which is uniquely determined by its Tutte polynomial. Hence the class $\operatorname{Ex}\left(C_{4}^{+}\right)$is also characterized by the Tutte polynomial. Another slightly less trivial example is given by $\operatorname{Ex}\left(C_{5}\right)$, whose 2-connected members are $K_{2, n}, n \geq 2$, and $K_{2, n}^{+}, n \geq 1$, (the complete bipartite graph $K_{2, n}$ with an extra edge joining the vertices of degree $n$ ). Again, $K_{2, n}$ and $K_{2, n}^{+}$are easily seen to be uniquely determined by their Tutte polynomial. All these examples of minor-closed classes characterized by the Tutte polynomial have the stronger property that each 2-connected member of the class is itself uniquely determined by the Tutte polynomial. This is not commonly the case for minor-closed classes. 
In [11] we can find two graphs with the same Tutte polynomial such that one of them has a cycle of length 8, while the longest cycle in the other one has length 7 . This shows that $\operatorname{Ex}\left(C_{8}\right)$ is not characterized by the Tutte polynomial. The example can be easily modified (by subdividing the edge $e$ in Figure 1 from [11]), to show that $\operatorname{Ex}\left(C_{k}\right)$ is not characterized by the Tutte polynomial when $k \geq 8$. We do not know whether this is the case for $\operatorname{Ex}\left(C_{6}\right)$ and $\operatorname{Ex}\left(C_{7}\right)$.

To conclude, we believe it is worth exploring further classes of graphs characterized by the Tutte polynomial, whether defined in terms of minors or otherwise.

\section{References}

[1] M. Bodirsky, O. Giménez, M. Kang, and M. Noy, Enumeration and limit laws for series-parallel graphs, European J. Combin. 28 (2007), 2091-2105.

[2] T. H. Brylawski, A combinatorial model for series-parallel networks, Trans. Amer. Math. Soc. 154 (1971), 1-22.

[3] T. H. Brylawski, A decomposition for combinatorial geometries, Trans. Amer. Math. Soc. 171 (1972), 235-282.

[4] T. Brylawski and J. Oxley, The Tutte polynomial and its applications. Matroid applications, 123-225, Encyclopedia Math. Appl., 40, Cambridge Univ. Press, Cambridge, 1992.

[5] G. Chartrand and F. Harary, Planar permutation graphs, Ann. Inst. H. Poincaré Sect. B (N.S.) 3 (1967), 433-438.

[6] G.A. Dirac, A property of 4-chromatic graphs and some remarks on critical graphs, J. London Math. Soc. 27 (1952), 85-92.

[7] D. Eppstein, Parallel recognition of series-parallel graphs, Inform. Comput. 98 (1992), 41-55

[8] C. Merino, A. de Mier and M. Noy, Irreducibility of the Tutte polynomial of a connected matroid, J. Combin. Theory Ser. B $\mathbf{8 3}$ (2001), 298-304.

[9] A. de Mier, Graphs and matroids determined by their Tutte polynomials. PhD thesis, Universitat Politècnica de Catalunya, 2003.

[10] A. de Mier and M. Noy, On matroids determined by their Tutte polynomials, Discrete Math., 302 (2005), 52-76.

[11] W. Schwärzler, Being Hamiltonian is not a Tutte invariant, Discrete Math. 91 (1991), 87-89.

[12] H. Whitney, Non-separable and planar graphs, Trans. Amer. Math. Soc. 34 (1932), 339-362 